\title{
Modelling Excited States of Weakly Bound Complexes with Density Functional Theory
}

\author{
Edward A. Briggs and Nicholas A. Besley* \\ School of Chemistry, University of Nottingham, University Park, \\ Nottingham, NG7 2RD, UK. \\ E-mail: nick.besley@nottingham.ac.uk
}

To be published in Physical Chemistry Chemical Physics:

DOI: $10.1039 / \mathrm{C} 3 \mathrm{CP} 55361 \mathrm{~B}$ 


\begin{abstract}
The binding within the ethene-argon and formaldehyde-methane complexes in the ground and electronically excited states is studied with equation of motion coupled cluster theory (EOM-CCSD), second-order Møller-Plesset perturbation theory (MP2) and density functional theory with dispersion corrections (DFT-D). Electronically excited states are studied within MP2 and Kohn-Sham DFT formalisms by exploiting a procedure called the maximum overlap method that allows convergence of the relevant self-consistent field equations to higher energy (or excited state) solutions. Potential energy curves computed using MP2 are in good agreement with the EOMCCSD calculations for both the valence and Rydberg excited states studied. For the DFT-D approach, B3LYP-D3/aug-cc-pVTZ calculations are found to be in agreement with EOM-CCSD for the ground and valence excited states. However, for the $\pi 3 \mathrm{~s}$ Rydberg state of ethene-argon and the n3s Rydberg state of formaldehyde-methane significant deviation is observed, and this disagreement with EOM-CCSD is present for a variety of DFT-D based approaches. Variation of the parameters within the D2 dispersion correction results in closer agreement with EOM-CCSD for the Rydberg states but demonstrates that a different parameterisation from the ground state is required for these states. This indicates that time-dependent density functional theory calculations based upon a DFT-D reference may be satisfactory for excitations to valence states, but will potentially be inaccurate for excitations to Rydberg states, or more generally states were the nature of the electron density is significantly different from the ground state.
\end{abstract}




\section{Introduction}

Kohn-Sham density functional theory (DFT) ${ }^{1}$ has emerged as the most widely used quantum chemical method, and is used routinely to study of a diverse range of problems, encompassing materials science and biological systems. A serious deficiency of DFT using common exchange-correlation functionals is its failure to describe long range van der Waals (dispersion) interactions accurately. ${ }^{2-4}$ For many problems in areas such as supramolecular chemistry and protein structure, these interactions can play a critically important role and should not be neglected. In the case of electronically excited states, such interactions would also be important in the description of the structure of an excited chromophore within a biological system. ${ }^{5}$ The poor description of dispersion interactions within DFT can be attributed to the assumption that the exchange-correlation functional is a functional of the local electron density or the gradient of the local electron density. Wave function based methods, such as second-order Møller-Plesset perturbation theory (MP2) and coupled cluster theory $(\operatorname{CCSD}(\mathrm{T}))$, can provide an accurate treatment of dispersion. ${ }^{6-8}$ However, there remains a clear need for an accurate treatment of van der Waals interactions in computationally less demanding methods that can be applied to study large systems. Consequently, incorporating dispersion within DFT is a very active area of research, and many different approaches have been developed.

One approach to incorporating dispersion within DFT is to parameterise functionals that do not contain an explicit dispersion term using systems where dispersion interactions are prominent. Examples of this approach are the $\mathrm{M} 05^{9}$ and $\mathrm{M} 06^{10}$ functionals, which have proven successful for modelling some dispersion bound complexes. Other approaches have included the development of effective core potentials to incorporate the effects of dispersion. ${ }^{11}$ Alternatively, it is possible to treat dispersion explicitly as part of the exchange-correlation functional, and for a more thorough overview the reader is referred to some recent reviews. ${ }^{12-14}$ An emerging class of methods are those that attempt to treat dispersion rigorously via non-local functionals. ${ }^{15-19}$ While these methods increase the computational cost, they do hold promise of an accurate treatment of dispersion that is less empirical in nature. The most common approach for incorporating dispersion forces within DFT calculations, 
and the one that is focused on here, is based upon the addition of an empirically based damped $-C_{6} R^{-6}$ term in so-called DFT-D methods. ${ }^{20-24}$ The total energy is expressed as

$$
E_{D F T-D}=E_{K S-D F T}+E_{d i s p}
$$

where $E_{K S-D F T}$ is the usual DFT energy according to the chosen functional, and $E_{\text {disp }}$ is the dispersion energy that is typically given by

$$
E_{\text {disp }}=-S_{6} \sum_{A}^{N} \sum_{B<A}^{N} \frac{C_{6}^{A B}}{C_{A B}^{6}} f_{d m p}\left(R_{A B}\right)
$$

$N$ is the number of atoms in the system, $C_{6}{ }^{A B}$ is the dispersion coefficient for atom pair $A B, s_{6}$ is a scaling factor and $R_{A B}$ is the distance between atoms $A$ and $B$. A damping function $f_{d m p}\left(R_{A B}\right)$ is also included to avoid double counting of electron correlation effects and the near-singularites as $R_{A B} \rightarrow 0$. Within this framework there is considerable scope to vary how the various parameters are optimised, in addition to defining the precise nature of the damping function and how the pairwise dispersion coefficients are determined from the respective atomic values. ${ }^{13}$ These different approaches are typically assessed by comparison with data from accurate wave function based calculations on model systems, and benchmark data sets have been established. ${ }^{7}$ Grimme proposed the following expressions ${ }^{24}$

$$
\begin{aligned}
& C_{6}^{A B}=\sqrt{C_{6}^{A} C_{6}^{B}} \\
& f_{d m p}\left(R_{A B}\right)=\frac{1}{1+\exp ^{-d\left(R_{A B} / R_{A B}^{0}-1\right)}}
\end{aligned}
$$

where $d$ is a global scaling parameter for the damping function, and $R_{A B}^{0}$ is the sum of the van der Waals radii of atoms $A$ and $B$. Although alternative expressions for determining these parameters have been proposed. ${ }^{25}$ This formalism has been developed further in the DFT-D3 method where the dispersion energy is given by

$$
E_{\text {disp }}=-\sum_{A}^{N} \sum_{B<A}^{N} \sum_{n=6,8,10 . .} s_{n} \frac{C_{n}^{A B}}{R_{A B}^{n}} f_{d m p}\left(R_{A B}\right)
$$


and accounts for higher order dispersion coefficients. An alternative damping function is used and the method can also incorporate a three body energy. ${ }^{26}$ Becke and Johnson have proposed a model in which the dispersion interaction is evaluated by considering the interaction between the instantaneous dipole moment of the exchange hole in one molecule and the induced dipole moment in another. ${ }^{27-30}$ This provides a mechanism to determine the dispersion coefficients from the electron density.

These methods have proven to be an important addition to DFT and are now used extensively. However, the application of these methods has nearly exclusively considered the interaction between molecules in the electronic ground state. The interaction within complexes where one component is in an electronically excited state is important in the context of spectroscopy in rare gas matrices and the condensed phase. Since the commonly used dispersion corrections are empirical, the underlying parameterisation using ground state data may not be suitable for an excited state that could have significantly different electronic structure. Previous work has considered the interaction between argon and nitric oxide in its ground $X^{2} \Pi$ and excited $\mathrm{A}^{2} \Sigma^{+}$states with DFT with a range of corrections for dispersion. ${ }^{31}$ For the ground state, the DFT based calculations were in good agreement with coupled cluster theory data, however none of the functionals gave satisfactory agreement for the excited state. More recently, Fukuda and Ehara studied electronic excitations in a free-base porphyrin-Ar $\mathrm{A}_{2}$ complex with symmetry-adapted cluster-configuration interaction (SAC-CI) and time-dependent density functional theory (TDDFT). ${ }^{32}$ In these calculations, TDDFT did not reproduce the observed shift to higher energy for the $1^{1} \mathrm{~B}_{3 \mathrm{u}}$ state but did correctly predict shifts to lower energy for the B-band states. In this paper, the performance of DFT-D methods in describing weakly bound complexes where one component is in an electronically excited state is assessed relative to EOM-CCSD and MP2 calculations using two model complexes, etheneargon and formaldehyde-methane, with different excited states. 


\section{Computational Details}

The molecular complexes and different excited states considered are illustrated in Figure 1. The ethene-argon complex is considered as a model of $\pi \rightarrow \pi^{*}$ transitions in unsaturated hydrocarbons. The argon atom is placed above the centre of mass of the ethene molecule. The interaction energy is computed as $r$ is varied, where $r$ corresponds to the perpendicular distance between the center of mass of ethene and the argon atom, no angular variations are considered. Along with the ground state, the two excited states of ethene arising from $\pi \rightarrow \pi^{*}$ and $\pi \rightarrow 3 \mathrm{~s}$ transitions are studied. For the formaldehyde-methane complex, the interaction energies for the ground state and excited states following $n \rightarrow \pi^{*}$ and $n \rightarrow 3$ s transitions are considered. In this complex, the methane molecule is placed directly above the carbon atom of $\mathrm{H}_{2} \mathrm{CO}$ perpendicular to the plane containing formaldehyde. For this complex, $r$ represents the distance between the two carbon atoms.

Potential energy curves have been computed for the ground and excited states using EOM-CCSD, MP2, DFT and DFT-D methods. All MP2 and DFT calculations were corrected for basis set superposition error using the counterpoise correction. ${ }^{33}$ EOMCCSD calculations were performed in conjunction with the aug-cc-pVDZ basis set. Additional CCSD and EOM-CCSD calculations to determine the binding energy with the larger aug-cc-pVTZ basis set were performed to assess the convergence of the calculated interaction energies with respect to the size of the basis set. These calculations were performed at the minimum separation according to the aug-ccpVDZ basis set, and full potential energy curves were not computed. For the MP2 and DFT calculations, excited states were determined by exploiting a scheme called the maximum overlap method (MOM) that allows the self-consistent-field procedure within an unrestricted Hartree-Fock (HF) or Kohn-Sham-DFT calculation to converge to give higher energy (excited state) solutions. ${ }^{34}$ This procedure has been applied to study the excited states in a variety of systems. ${ }^{35-37}$ Recently, it was shown that rovibrational spectrum for the $\widetilde{C} \leftarrow \widetilde{X}$ transition in the NO-Ar complex simulated using MP2 potential energy surfaces computed using this method were in excellent agreement with experiment, demonstrating the accuracy of the approach for systems 
of a similar nature when used in conjunction with MP2. ${ }^{38}$ For the excited state calculations presented here, the complex is initially considered at a large separation with the molecular geometries optimised at the MP2/cc-pVTZ level. The ground state orbitals with the occupancies altered to reflect the relevant excited state were used as the initial guess for the MOM calculation. Subsequently, the intermolecular separation was reduced in a stepwise manner, and for each calculation the converged orbitals from the previous step were used as the initial guess. By following this procedure, the MOM approach was able to prevent any variational collapse to the ground state. For the MP2 calculations, the correlation energy was extrapolated to the complete basis set (CBS) limit using a two-point extrapolation relationship ${ }^{39}$ based upon the aug-ccpVDZ and aug-cc-pVTZ basis sets. Alternative methods such as the algebraic diagrammatic construction (ADC) scheme could be used to study the excited states within a wavefunction based formalism. However, this approach would be expected to give results that are similar to EOM-CCSD and MOM-MP2 methods.

DFT calculations have been performed with the B3LYP ${ }^{40,41}$ and $\mathrm{PBE}^{42,43}$ exchangecorrelation functionals in conjunction with the D2, D3 and XDM dispersion corrections and with the aug-cc-pVTZ basis set. Standard values of the scaling factors for B3LYP and PBE were used in the D2 and D3 dispersion corrections, ${ }^{24,26}$ and the 3-body interaction term in the D3 correction was not applied. For the XDM correction, Becke's damping function including only the $C_{6}$ term was used. In order to obtain smooth potential energy curves a 250 Euler-Maclaurin radial integration grid with 590 point Lebdev angular grid was used. All calculations were performed with the Q-Chem software package. ${ }^{44}$

\section{Results and Discussion}

EOM-CCSD and MP2

Figures 2 and 3 show the computed EOM-CCSD, MP2/CBS, B3LYP and B3LYP-D3 potential energy curves for the ethene-argon and formaldehyde-methane complexes, with the binding energies and geometries of the minima given in Table 1. Initially, 
we will discuss the EOM-CCSD and MP2 curves. The ground state of ethene-argon is predicted to be bound by $-595 \mu \mathrm{E}_{\mathrm{h}}$ with a separation of $r_{e}=4.1 \AA$ at the CCSD/aug-ccpVDZ level, with the binding energy increasing to $-763 \mu \mathrm{E}_{\mathrm{h}}$ with the larger aug-ccpVTZ basis set. At the MP2 level a binding energy of $-681 \mu \mathrm{E}_{\mathrm{h}}$ and $r_{e}=3.8 \AA$ are obtained, which are in reasonable agreement with the CCSD values. The focus of this study is the interaction when the molecule is an excited state and if a comparable degree of accuracy is obtained for excited states as is observed for the ground state. If ethene is excited to the $\pi \pi^{*}$ excited state, there is a large increase in the strength of the interaction, with the binding energy increasing to $-2454 \mu \mathrm{E}_{\mathrm{h}}$ and $r_{e}$ decreasing to 3.1 A. This binding energy changes to $-2289 \mu \mathrm{E}_{\mathrm{h}}$ for the larger basis set. A significant increase in the binding energy compared to the ground state is also predicted by the MP2 calculations, with a computed binding energy of $-2890 \mu \mathrm{E}_{\mathrm{h}}$ and a separation of $3.3 \AA$. The potential energy curve for the $\pi 3 \mathrm{~s}$ state is more complex with two minima. EOM-CCSD predicts these minima to lie at $3.1 \AA$ and $6.2 \AA$ with binding energies of $-284 \mu \mathrm{E}_{\mathrm{h}}$ and $-124 \mu \mathrm{E}_{\mathrm{h}}$, respectively. The MP2 calculations give a potential energy curve with a similar shape, although the depth of the minimum at short range is underestimated compared to the coupled cluster calculations. The observed shape of this potential energy curve suggests that there may be an avoided crossing. For ethene this is unlikely to be the case since the next excited state is a Rydberg $\pi 3 p$ state that lies about $0.5 \mathrm{eV}$ higher in energy. Furthermore, there would also be a change in electronic state as $r$ is increased. Within the MOM formalism the nature of the excited state is explicitly maintained during the calculations, which excludes the possibility of a change of state. However, for a Rydberg state that by its nature is more diffuse a minimum at larger $r$ values is not surprising.

The ground state of the formaldehyde-methane complex is relatively strongly bound, with a binding energy of $-1151 \mu \mathrm{E}_{\mathrm{h}}$ and $r_{e}=3.6 \AA$. For both of the excited states the binding energy at the minimum decreases, particularly for the Rydberg $n 3 \mathrm{~s}$ state. Similar to the $\pi 3 \mathrm{~s}$ state of ethene-argon, the potential energy curve for the $n 3 \mathrm{~s}$ state shows two minima, a deeper one at $r_{e}=6.0 \AA$ and a more shallow minimum at $r_{e}=3.6$ $\AA$. The potential energy curves computed with MP2 show similar features. The binding energy for the ground state is predicted to be $80 \mathrm{~cm}^{-1}$ greater compared to CCSD, while for the $\pi^{*}$ state the predicted $r_{e}$ is in good agreement with EOM-CCSD but the binding energy is significantly smaller. For the n3s state two minima are found with MP2, but like ethene-argon the depth of the minimum at short range is 
larger than the one at larger $r$.

While there a some discrepancies between the precise values of the binding energies, the EOM-CCSD and MP2 calculations do provide a reasonably consistent picture of the potential energy curves of the different electronic states. For the ethene-argon complex the binding energy in the ground state is about $600-700 \mu \mathrm{E}_{\mathrm{h}}$ with a large, approximately four fold increase, in the binding energy for the $\pi \pi^{*}$ state. For the $\pi 3 \mathrm{~s}$ state a minimum at short-range $(\sim 3.1 \AA)$ and long-range $(\sim 6.2 \AA)$ are observed. The ground state of formaldehyde-methane is more strongly bound with a binding energy of $\sim 1200 \mu \mathrm{E}_{\mathrm{h}}$ and there is a decrease in the strength of the interaction for the $n \pi^{*}$ state. Again, for the Rydberg state two minima are observed. The minimum at larger $r$ has a binding energy of $\sim 250 \mu \mathrm{E}_{\mathrm{h}}$, while there is disagreement over the binding energy for the minimum at shorter $r$.

\section{DFT and DFT-D}

Figures 2 and 3 also show the computed potential energy curves for DFT and DFT-D calculations with the B3LYP functional, D3 dispersion correction using the aug-ccpVTZ basis set. For the ethene-argon system, no binding is predicted for any of the states when dispersion is not included. It is interesting to note that for the $\pi 3 \mathrm{~s}$ state, the distinct shape with a minimum at $3.1 \AA$ and maximum at $\sim 4$ that is present in the MP2 and EOM-CCSD calculations is evident. This suggests that for this state, the minimum observed at larger $r$ arises from dispersion. For this complex the binding arises from the dispersion correction, and with the inclusion of dispersion the resulting potential energy curves for the ground state and $\pi \pi^{*}$ state are in qualitative agreement with the wave function based calculations. The predicted values of $r_{e}$ are in close agreement with the wave function based calculations, but the depths of the minima are underestimated by about $20-30 \%$.

In contrast, for the $\pi 3 \mathrm{~s}$ Rydberg state the inclusion of dispersion leads to significant deviation from both EOM-CCSD and MP2 calculations. The resulting curve has a 
deep minimum at $3.0 \AA$ and shows a significantly larger deviation from the EOMCCSD and MP2 results than the B3LYP with no dispersion curve. A similar picture emerges for the formaldehyde-methane system. Binding is observed once the dispersion correction is included, and there is reasonable agreement with the wave function based results for the ground state and $n \pi^{*}$ states. However, for the Rydberg state the binding energy is much too large. These results show that while the dispersion correction works quite well for the valence excited states, its performance for the Rydberg excited states is much poorer. This suggests that the electronic structure of the Rydberg excited state is sufficiently different from that of the ground state to make the parameterisation of the dispersion correction no longer appropriate.

B3LYP-D3 represents only one of the many possible combinations of exchangecorrelation functional and dispersion correction. Figure 4 shows computed potential energy curves for alternative dispersion corrections and exchange-correlation functionals, with the computed properties of the minima given in Table 2. The potential energy curves computed using B3LYP-D2 are very similar to B3LYP-D3, with little variation in the location of the minima but for all three states the strength of binding is predicted to be weaker with the D2 correction. With the XDM dispersion correction the binding is further weakened and significantly underestimated relative to EOM-CCSD. For the PBE exchange-correlation functional distinct minima are observed for all three states when no dispersion correction is applied. This is in contrast to B3LYP where no binding is predicted. In fact for the ground state the depth of the minima for PBE is comparable to B3LYP-XDM albeit at a larger value of $r$. For the $\pi 3 \mathrm{~s}$ state, the curve for B3LYP resembles those from EOM-CCSD and MP2, while the PBE curve is significantly different. This suggests that B3LYP represents a better choice of underlying exchange-correlation functional. PBE in conjunction with D3 and XDM corrections overestimate the value for the equilibrium separation and underestimate the binding compared to EOM-CCSD for the ground and $\pi \pi^{*}$ states. Crucially, while many of the DFT-D calculations provide a reasonable description for the ground and $\pi \pi^{*}$ states of the ethene-argon complex, none are correct the $\pi 3 \mathrm{~s}$ state. This is consistent with earlier work on the excited $\mathrm{A}^{2} \Sigma^{+}$ Rydberg state of the NO-Ar complex, where poor agreement with high level wave 
function based calculations was observed. ${ }^{31}$

\section{Variation of the Dispersion Correction}

The discrepancy for the $\pi 3 \mathrm{~s}$ state of ethene-argon is illustrated clearly in Figure 5 which shows the difference between the EOM-CCSD curve and the B3LYP curve without the addition of dispersion. This indicates how a dispersion correction for this state would appear in order to bring the B3LYP curve into agreement with EOMCCSD. This 'correct' dispersion contribution has a minimum of approximately -525 $\mu \mathrm{E}_{\mathrm{h}}$ at $3.2 \AA$. Also plotted is the actual dispersion contribution as given by the $\mathrm{D} 2$ dispersion correction with default parameters. At long range the D2 dispersion correction is too small, but more significantly the minimum at $3.2 \AA$ is much too deep. This indicates that the parameterisation of the dispersion correction for the ground state is not appropriate for the excited Rydberg state.

In order to explore how the dispersion contribution varies as the key parameters in the D2 correction are changed, the dispersion contributions arising from reducing and increasing the $\mathrm{C}_{6}$ coefficients and Van der Waals radii are shown. Reducing the $\mathrm{C}_{6}$ coefficients for carbon and hydrogen reduces the depth of the minimum, but a significant deviation from the correct dispersion curve remains. Increasing the van der Waals radii of carbon and hydrogen has a greater effect and the resulting dispersion contribution is considerably closer to the correct one. This has some physical basis since Rydberg states are known to have a greater spatial extent than valence states, however, such a large increase of a factor of two is hard to justify based upon analysis of the electron densities.

Figure 6 shows the resulting potential energy curves for the $\pi 3 \mathrm{~s}$ state of ethene-argon and $\mathrm{n} 3 \mathrm{~s}$ state of formaldehyde-methane with modified van der Waals radii. For the formaldehyde-methane complex the van der Waals radii of the atoms in formaldehyde are increased by a factor of two, while the van der Waals radii for the methane atoms 
are not changed from their default value. For both of these states, the resulting potential energy curve with the modified D2 parameters are much closer to the EOMCCSD and MP2 curves compared to the potential energy curve with default parameters. For the ethene-argon complex the new B3LYP-D2 curve is in excellent agreement with the wave function based methods. A larger deviation is observed for formaldehyde-methane, but the resulting curve is comparable with that from MP2. This demonstrates that the nature of the EOM-CCSD curves can be captured by the D2 correction, and clearly with a more comprehensive re-parameterisation more accurate curves would result.

Finally, we consider the $\mathrm{NO}\left(\mathrm{A}^{2} \Sigma^{+}\right)$-Ar complex, wherein an argon atom is interacting with the first excited Rydberg state of NO. This complex has been studied previously where it was shown that DFT-D methods do not provide a good description of the interaction potential. ${ }^{31}$ In particular, B3LYP-D3 calculations predicted a minimum energy structure with a Ar-N-O angle of $30^{\circ}$, whereas high level calculations and

experiment find the angle to be $0^{\circ} \cdot{ }^{45-47} \mathrm{~A}$ further shallow minimum is predicted for a Ar-N-O angle of $180^{\circ}$, in agreement with experiment and previous calculations. ${ }^{45-47}$ This is illustrated in Figure 7 that shows the interaction potential at the B3LYP-D2 level, which closely resembles the B3LYP-D3 one. Also shown is an analogous potential energy surface computed with B3LYP-D2 with modified (increased by a factor of 2 for nitrogen and oxygen) van der Waals radii. This potential does correctly describe the minimum energy structure to be linear with $\theta=0^{\circ}$, while there is very little variation in the position and depth of the minimum for Ar on the oxygen side of NO. However, the depth of the minimum is underestimated with a calculated value of $23.2 \mathrm{~cm}^{-1}$ compared to the experimental values of -105 to $-120 \mathrm{~cm}^{-1}$. ${ }^{45-47}$

\section{Conclusions}

The description of the ground and excited states of the two weakly bound complexes, ethene-argon and formaldehyde-methane, with DFT-D based methods has been assessed relative to EOM-CCSD and MP2 calculations. In the ethene-argon complex, excitation of ethene to give the $\pi \pi^{*}$ state leads to an increase in the strength of binding, while the complex is more weakly bound on excitation to the $\pi 3$ s state. For 
the formaldehyde-methane complex, both excitation to $n \pi^{*}$ and $n 3 s$ states lead to weaker binding compared to the ground state.

DFT-D calculations with the dispersion component modelled according to the empirical scheme of Grimme reproduce the potential energy curves for the ground and valence excited states with a good level of accuracy. For the $\pi 3 \mathrm{~s}$ and n3s Rydberg states, B3LYP-D3 calculations predict the binding to be too strong. For the $\pi 3$ s state of ethene-argon this is also observed with a variety of DFT-D approaches. This is despite the uncorrected B3LYP potential energy curve providing a reasonable description of the EOM-CCSD and MP2 curves. Modification of the $\mathrm{C}_{6}$ and van der Waals radii within the D2 correction shows that a significantly improved agreement with the wave function based methods is achieved if the atomic van der Waals radii are increased. This demonstrates that these states can be described within the framework of a mathematically simple dispersion term but that the appropriate parameters will be different from the ground state. This clearly has consequences for describing the excited states of such systems with TDDFT based upon Kohn-Sham DFT-D calculations, which assume that the dispersion in the excited state is the same as in the ground (reference) state. Clearly, for the B3LYP and PBE functionals considered here, excitations to Rydberg states would require a state specific dispersion correction. This would be difficult to realise within current TDDFT approaches.

\section{Acknowledgements}

We thank the University of Nottingham High Performance Computing facility for providing computer resources and a studentship for EAB.

\section{References}

${ }^{1}$ W. Kohn and L. J. Sham, Phys. Rev., 1965, 140, A1133-A1138.

${ }^{2}$ S. Kristyan and P. Pulay, Chem. Phys. Lett., 1994, 229, 175-180. 
${ }^{3}$ P. Hobza, J. Sponer and T. Reschel, J. Comput. Chem., 1995, 16, 1315-1325.

${ }^{4}$ J. M. Perez-Jorda and A. D. Becke, Chem. Phys. Lett., 1995, 233, 134-137.

${ }^{5}$ D. Robinson, N. A. Besley P. O’Shea and J. D. Hirst, J. Phys. Chem. B, 2011, 115, 4160-4167.

${ }^{6}$ M. O. Sinnokrot and C. D. Sherrill, J. Phys. Chem. A, 2006, 110, 10656-10668.

${ }^{7}$ P. Jurecka, J. Sponer, J. Cerny and P. Hobza, Phys. Chem. Chem. Phys., 2006, 8, 1985-1993.

${ }^{8}$ C. A. Morgado, P. Jurecka, D. Svozil, P. Hobza and J. Sponer, Phys. Chem. Chem. Phys., 2010, 12, 3522-3534.

${ }^{9}$ Y. Zhao, N. E. Schultz and D. G. Truhlar, J. Chem. Theory Comput., 2006, 2, $364-$ 382.

${ }^{10}$ Y. Zhao and D. G. Truhlar, Theor. Chem. Acc., 2008, 120, 215-241.

${ }^{11}$ O. A. von Lilienfeld, I. Tavernelli, U. Röthlisberger and D. Sebastiani, Phys. Rev. Lett., 2004, 93, 153004.

${ }^{12}$ S. Grimme, J. Antony, T. Schwabe and C. Mück-Lichtenfeld, Org. Biomol. Chem., 5, 741-758.

${ }^{13}$ M. E. Foster and K. Sohlberg, Phys. Chem. Chem. Phys., 2010, 12, 307-322.

${ }^{14}$ S. Grimme, WIREs Comput. Mol. Sci., 2011, 1, 211-228.

${ }^{15}$ M. Dion, H. Rydberg, E. Schröder, D. C. Langreth, and B. I. Lundqvist, Phys. Rev. Lett., 2004, 92, 246401.

${ }^{16}$ S. Grimme, J. Chem. Phys., 2006, 124, 034108. 
${ }^{17}$ O. A. Vydrov and T. Van Voorhis, Phys. Rev. Lett., 2009, 103, 063004.

${ }^{18}$ A. Tkatchenko and M. Scheffler, Phys. Rev. Lett., 2009, 102, 073005.

${ }^{19}$ N. Marom, A. Tkatchenko, M. Rossi, V. V. Gobre, O. Hod, M. Scheffler and L. Kronik, J. Chem. Theory Comput., 2011, 7, 3944-3951.

${ }^{20}$ M. Elstner, P. Hobza, T. Frauenheim, S. Suhai and E. Kaxiras, J. Chem. Phys., 2001, 114, 5149-5155.

${ }^{21}$ Q. Wu and W. Yang, J. Chem. Phys., 2001, 115, 8748-8757.

${ }^{22}$ U. Zimmerli, M. Parrinello and P. Koumoutsakos, J. Chem. Phys., 2004, 120, $2693-$ 2699.

${ }^{23}$ S. Grimme, J. Comput. Chem., 2004, 25, 1463-1473.

${ }^{24}$ S. Grimme, J. Comput. Chem., 2006, 27, 1787-1799.

${ }^{25}$ P. Jurecka, J. Cerny, P. Hobza and D. Salahub, J. Comput. Chem., 2007, 28, 555569.

${ }^{26}$ S. Grimme, J. Antony, S. Ehrlich and H. Krieg, J. Chem. Phys., 2010, 132, 154104.

${ }^{27}$ A. D. Becke and E. R. Johnson, J. Chem. Phys., 2005, 122, 154104.

${ }^{28}$ E. R. Johnson and A. D. Becke, J. Chem. Phys., 2005, 123, 024101.

${ }^{29}$ A. D. Becke and F. O. Kannemann, Can. J. Chem., 2010, 88, 1057-1062.

${ }^{30}$ F. O. Kannemann and A. D. Becke, J. Chem. Theory Comput., 2010, 6, 1081-1088.

${ }^{31}$ O. V. Ershova and N. A. Besley, J. Chem. Phys., 2012, 136, 244313. 
${ }^{32}$ R. Fukuda and M. Ehara, J. Chem. Phys., 2013, 139, 074303.

${ }^{33}$ S. F. Boys and F. Bernardi, Mol. Phys., 1970, 19, 553-566.

${ }^{34}$ A. T. B. Gilbert, N. A. Besley, and P. M. W. Gill, J. Phys. Chem. A., 2008, 112, 13164-13171.

${ }^{35}$ N. A. Besley, A. T. B. Gilbert and P. M. W. Gill, J. Chem. Phys., 2009, 130, 124308 .

${ }^{36}$ O. V. Ershova and N. A. Besley, Chem. Phys. Lett., 2011, 513, 179.

${ }^{37}$ M. W. D. Hanson-Heine, M. W. George and N. A. Besley, J. Chem. Phys., 2013, 138, 064101.

${ }^{38}$ O. V. Ershova, J. Klos, J. P. Harris, A. M. Gardner, V. M. Tamé-Reyes, A. Andrejava, M. H. Alexander, N. A. Besley, and T. G. Wright, J. Chem. Phys., 2013, 138, 214313.

${ }^{39}$ A. Halkier, T. Helgaker, P. Jørgensen, W. Klopper, H. Koch, J. Olsen and A. K. Wilson, Chem. Phys. Lett., 1998, 286, 243-252.

${ }^{40}$ A. D. Becke, J. Chem. Phys., 1993, 98, 5648-5652.

${ }^{41}$ P. J. Stephens, F. J. Devlin, C. F. Chabalowski and M. J. Frisch, J. Phys. Chem., 1994, 98, $11623-11627$.

42 J. P. Perdew, K. Burke and M. Ernzerhof, Phys. Rev. Lett., 1996, 77, 3865-3868.

${ }^{43}$ J. P. Perdew, K. Burke and M. Ernzerhof, Phys. Rev. Lett., 1997, 78, 1396.

${ }^{44}$ Y. Shao, L. Fusti Molnar, Y. Jung, J. Kussmann, C. Ochsenfeld, S. T. Brown, A. T. B. Gilbert, L. V. Slipchenko, S. V. Levchenko, D. P. O'Neill, R. A. DiStasio Jr., R. C. 
Lochan, T. Wang, G. J. O. Beran, N. A. Besley, J. M. Herbert, C. Y. Lin, T. Van Voorhis, S. H. Chien, A. Sodt, R. P. Steele, V. A. Rassolov, P. E. Maslen, P. P. Korambath, R. D. Adamson, B. Austin, J. Baker, E. F. C. Byrd, H. Daschel, R. J. Doerksen, A. Dreuw, B.D. Dunietz, A. D. Dutoi, T. R. Furlani, S. R. Gwaltney, A. Heyden, S. Hirata, C. P. Hsu, G. Kedziora, R. Z. Khaliullin, P. Klunzinger, A. M. Lee, M. S. Lee, W. Z. Liang, I. Lotan, N. Nair, B. Peters, E. I. Proynov, P. A. Pieniazek, Y. M. Rhee, J. Ritchie, E. Rosta, C. D. Sherrill, A. C. Simmonett, J. E. Subotnik, H. L. Woodcock III, W. Zhang, A. T. Bell, A. K. Chakraborty, D. M. Chipman, F. J. Keil, A. Warshel, W. J. Hehre, H. F. Schaefer III, J. Kong, A. I. Krylov, P. M.W. Gill and M. Head-Gordon, Phys. Chem. Chem. Phys., 2006, 8, 31723191.

${ }^{45}$ K. Tsuji, K. Shibuya and K. Obi, J. Chem. Phys., 1994, 100, 5441-5447.

${ }^{46}$ J. Klos, M. H. Alexander, R. Hernandez-Lamoneda and T. G. Wright, J. Chem. Phys., 2008, 129, 244303.

${ }^{47}$ H. L. Holmes-Ross and W. D. Lawrence, J. Chem. Phys., 2011, 135, 014302. 
Table 1: Computed binding energies (in $\mu \mathrm{E}_{\mathrm{h}}$ ) of the complexes with the minimum energy separation (in $\AA$ ) in parenthesis.

\begin{tabular}{|c|c|c|c|c|c|}
\hline Complex & State & $\begin{array}{l}(\mathrm{EOM})-\mathrm{CCSD} / \\
\text { aug-cc-pVDZ }\end{array}$ & $\begin{array}{l}\text { MP2/ } \\
\text { CBS }\end{array}$ & $\begin{array}{c}\text { B3LYP/ } \\
\text { aug-cc-pVTZ }\end{array}$ & $\begin{array}{l}\text { B3LYP-D3/ } \\
\text { aug-cc-pVTZ }\end{array}$ \\
\hline \multirow[t]{3}{*}{$\mathrm{C}_{2} \mathrm{H}_{4}-\mathrm{Ar}$} & g.s. & $\begin{array}{c}-595.2 \\
\left(r_{e}=4.1\right)\end{array}$ & $\begin{array}{c}-680.8 \\
\left(r_{e}=3.8\right)\end{array}$ & no binding & $\begin{array}{c}-459.3 \\
\left(r_{e}=3.9\right)\end{array}$ \\
\hline & $\pi \pi^{*}$ & $\begin{array}{l}-2453.6 \\
\left(r_{e}=3.1\right)\end{array}$ & $\begin{array}{l}-2889.7 \\
\left(r_{e}=3.3\right)\end{array}$ & no binding & $\begin{array}{l}-1780.1 \\
\left(r_{e}=3.3\right)\end{array}$ \\
\hline & $\pi 3 \mathrm{~s}$ & $\begin{array}{c}-284.1 \\
\left(r_{e}=3.1\right) \\
-123.8 \\
\left(r_{e}=6.2\right)\end{array}$ & $\begin{array}{c}107.2 \\
\left(r_{e}=3.2\right) \\
-154.4 \\
\left(r_{e}=6.0\right)\end{array}$ & no binding & $\begin{array}{l}-2308.4 \\
\left(r_{e}=3.0\right)\end{array}$ \\
\hline \multirow[t]{3}{*}{$\mathrm{H}_{2} \mathrm{CO}-\mathrm{CH}_{4}$} & g.s. & $\begin{array}{l}-1150.8 \\
\left(r_{e}=3.6\right)\end{array}$ & $\begin{array}{l}-1230.7 \\
\left(r_{e}=3.4\right)\end{array}$ & $\begin{array}{l}<-10.1 \\
\left(r_{e}>8.0\right)\end{array}$ & $\begin{array}{l}-1000.3 \\
\left(r_{e}=3.6\right)\end{array}$ \\
\hline & $\mathrm{n} \pi^{*}$ & $\begin{array}{c}-854.0 \\
\left(r_{e}=3.9\right)\end{array}$ & $\begin{array}{c}-444.4 \\
\left(r_{e}=3.8\right)\end{array}$ & $\begin{array}{c}<-4.6 \\
\left(r_{e}>8.0\right)\end{array}$ & $\begin{array}{c}-513.3 \\
\left(r_{e}=3.9\right)\end{array}$ \\
\hline & $\mathrm{n} 3 \mathrm{~s}$ & $\begin{array}{c}138.7 \\
\left(r_{e}=3.6\right) \\
-258.0 \\
\left(r_{e}=6.0\right)\end{array}$ & $\begin{array}{c}-836.8 \\
\left(r_{e}=3.1\right) \\
-232.9 \\
\left(r_{e}=5.4\right)\end{array}$ & $\begin{array}{c}-20.6 \\
\left(r_{e}=7.9\right)\end{array}$ & $\begin{array}{l}-2003.7 \\
\left(r_{e}=3.2\right)\end{array}$ \\
\hline
\end{tabular}


Table 2: Computed binding energies (in $\mu \mathrm{E}_{\mathrm{h}}$ ) and minimum energy separations (in $\AA$ ) for ethene-argon with different functionals.

\begin{tabular}{|l|c|c|c|}
\hline \multicolumn{1}{|c|}{ Method } & Ground state & $\pi \pi^{*}$ state & $\pi 3$ s state \\
\hline B3LYP-D3 & $-459.3\left(r_{e}=3.91\right)$ & $-1780.1\left(r_{e}=3.25\right)$ & $-2308.4\left(r_{e}=3.03\right)$ \\
\hline B3LYP-D2 & $-289.4\left(r_{e}=3.89\right)$ & $-1605.6\left(r_{e}=3.25\right)$ & $-2081.8\left(r_{e}=3.06\right)$ \\
\hline B3LYP-XDM & $-113.2\left(r_{e}=4.22\right)$ & $-845.7\left(r_{e}=3.35\right)$ & $-1351.2\left(r_{e}=2.94\right)$ \\
\hline PBE & $-112.8\left(r_{e}=4.77\right)$ & $-299.5\left(r_{e}=4.41\right)$ & $-658.3\left(r_{e}=3.36\right)$ \\
\hline PBE-D3 & $-587.1\left(r_{e}=4.12\right)$ & $-1421.2\left(r_{e}=3.53\right)$ & $-3091.1\left(r_{e}=3.16\right)$ \\
\hline PBE-XDM & $-390.6\left(r_{e}=4.39\right)$ & $-787.1\left(r_{e}=3.85\right)$ & $-1849.7\left(r_{e}=3.20\right)$ \\
\hline (EOM)-CCSD & $-762.9\left(r_{e}=4.1\right)$ & $-2288.6\left(r_{e}=3.1\right)$ & $-628.3\left(r_{e}=3.1\right)$ \\
\hline
\end{tabular}




\section{Figure Captions}

Figure 1: The molecular orbitals involved in the electronic transitions. (a) ethene-Ar and (b) formaldehyde-methane.

Figure 2: Computed potential energy curves for the ground and excited states of the ethene-argon complex.

Figure 3: Computed potential energy curves for the ground and excited states of the formaldehyde-methane complex.

Figure 4: Computed DFT/aug-cc-pVTZ potential energy curves for the ground and excited states of the ethene-argon complex.

Figure 5: Comparison of the correct dispersion contribution and D2 dispersion corrections with altered parameters.

Figure 6: Computed potential energy curves for the $\pi 3 \mathrm{~s}$ state of ethene-argon and $\mathrm{n} 3 \mathrm{~s}$ state of formaldehyde-methane. Black line: EOM-CCSD/aug-cc-pVDZ, broken black line: MP2/CBS, blue line: B3LYP-D2 with default parameters, red line: B3LYP-D2 with increased van der Waals radii.

Figure 7: Computed B3LYP-D2/aug-cc-pVTZ potential energy surfaces for $\mathrm{NO}\left(\mathrm{A}^{2} \Sigma^{+}\right)$-Ar with default D2 parameters (left) and modified D2 parameters (right). $\mathrm{R}$ is the distance between the NO centre of mass and argon, and $\theta$ is the angle between the NO molecular axis and the line connecting argon and the NO centre of mass. The nitrogen atom is in blue, oxygen in red and argon in green. 
Figure 1: The molecular orbitals involved in the electronic transitions. (a) etheneargon and (b) formaldehyde-methane.

(a)

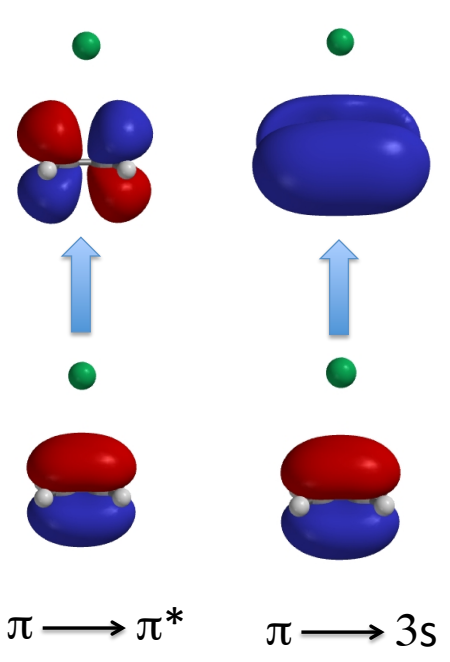

(b)<smiles>CC1CCCCC1</smiles>
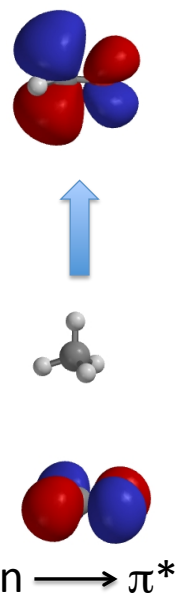
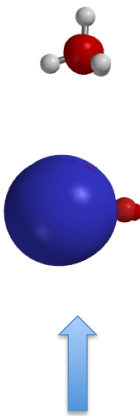

40

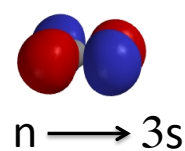


Figure 2: Computed potential energy curves for the ground and excited states of the ethene-argon complex.
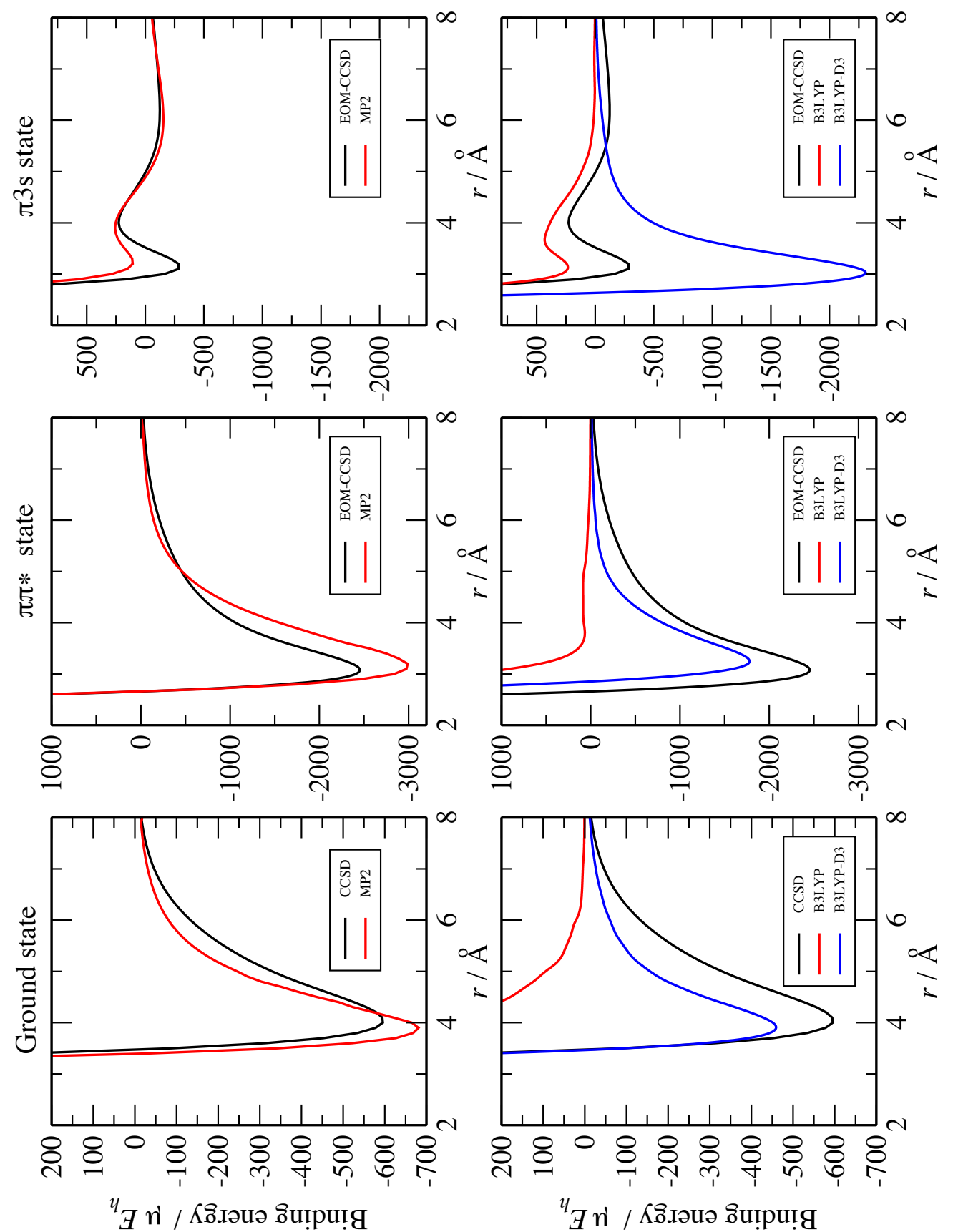
Figure 3: Computed potential energy curves for the ground and excited states of the formaldehyde-methane complex.
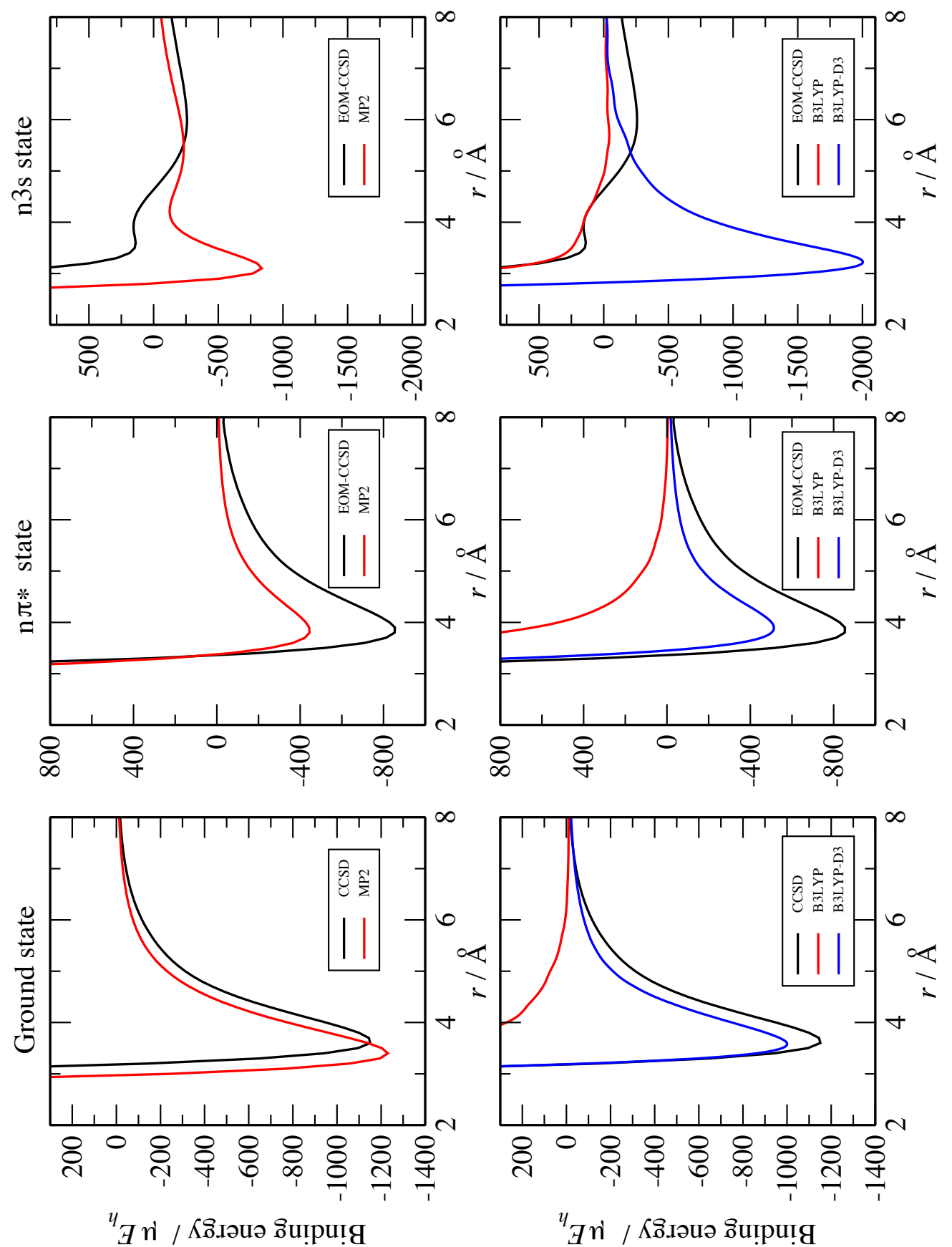
Figure 4: Computed DFT/aug-cc-pVTZ potential energy curves for the ground and excited states of the ethene-argon complex.

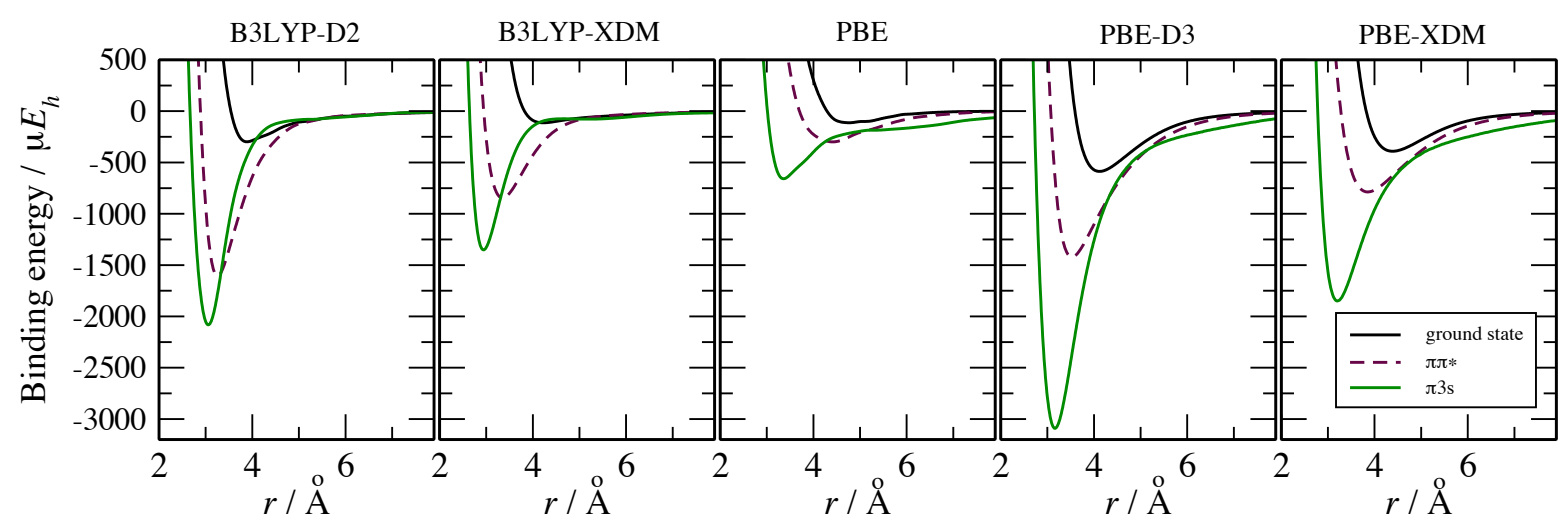


Figure 5: Comparison of the correct dispersion contribution and D2 dispersion corrections with altered parameters.
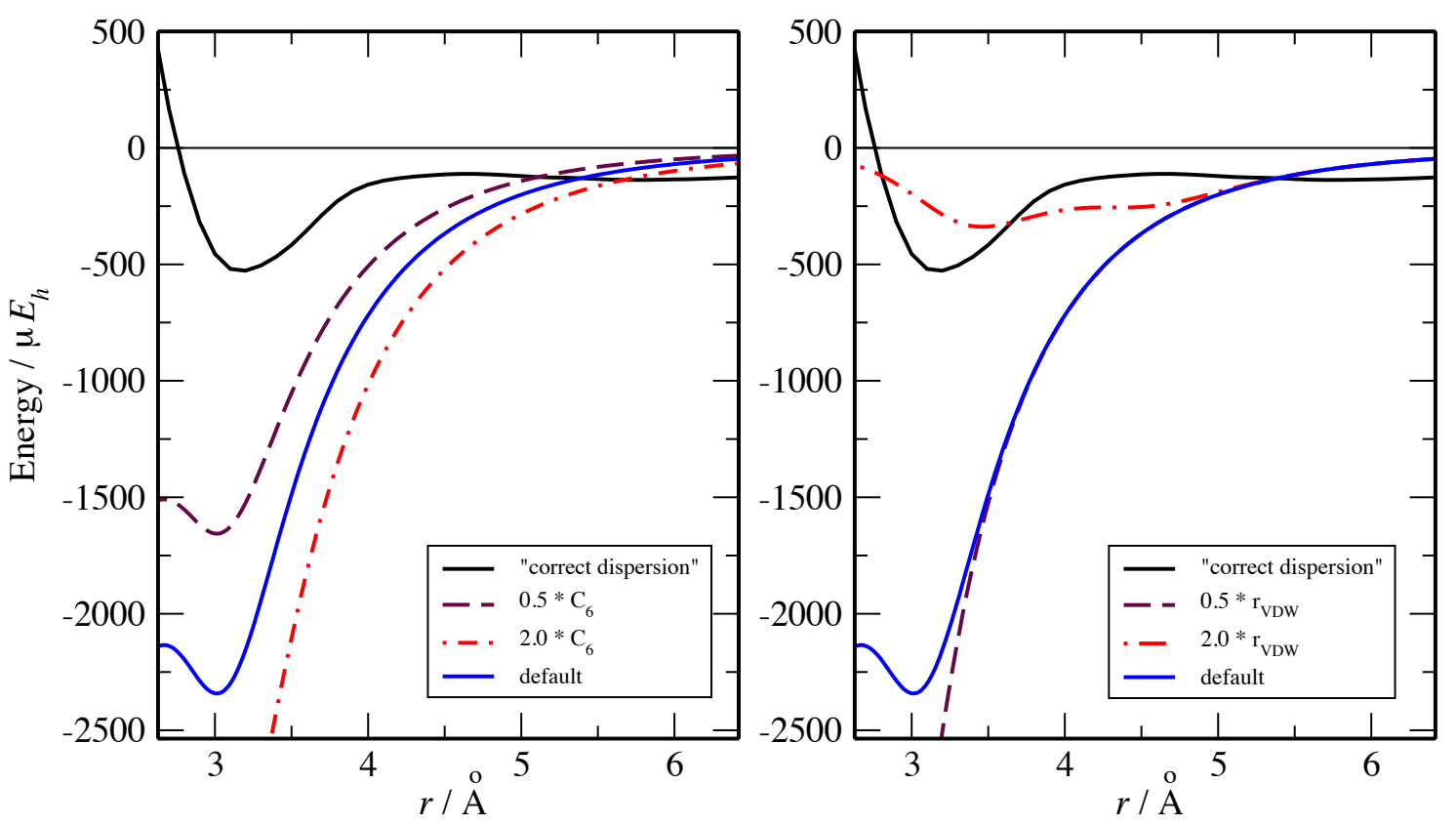
Figure 6: Computed potential energy curves for the $\pi 3 \mathrm{~s}$ state of ethene-argon and $\mathrm{n} 3 \mathrm{~s}$ state of formaldehyde-methane. Black line: EOM-CCSD/aug-cc-pVDZ, broken black line: MP2/CBS, blue line: B3LYP-D2 with default parameters, red line: B3LYP-D2 with increased van der Waals radii.
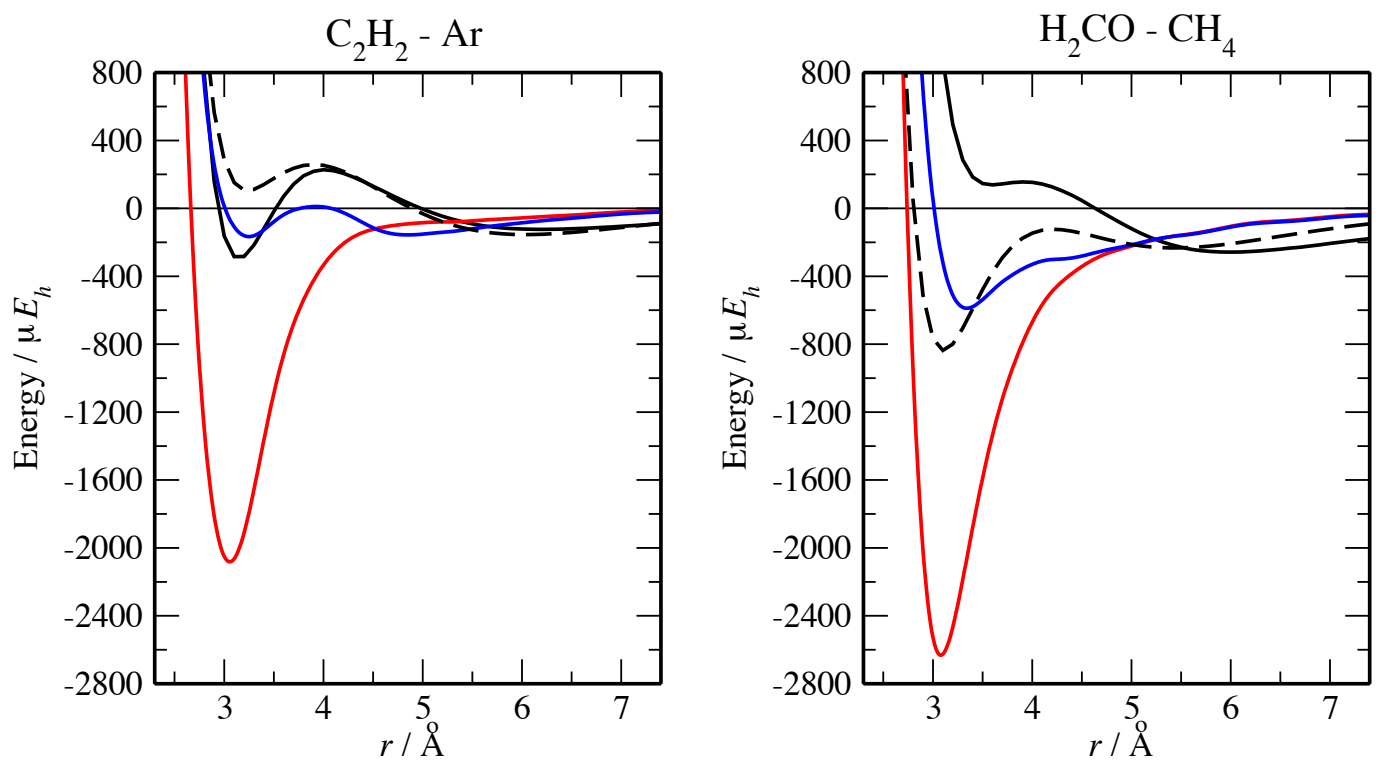
Figure 7: Computed B3LYP-D2/aug-cc-pVTZ potential energy surfaces for $\mathrm{NO}\left(\mathrm{A}^{2} \Sigma^{+}\right)$-Ar with default D2 parameters (left) and modified D2 parameters (right). $\mathrm{R}$ is the distance between the NO centre of mass and argon, and $\theta$ is the angle between the NO molecular axis and the line connecting argon and the NO centre of mass. The nitrogen atom is in blue, oxygen in red and argon in green.
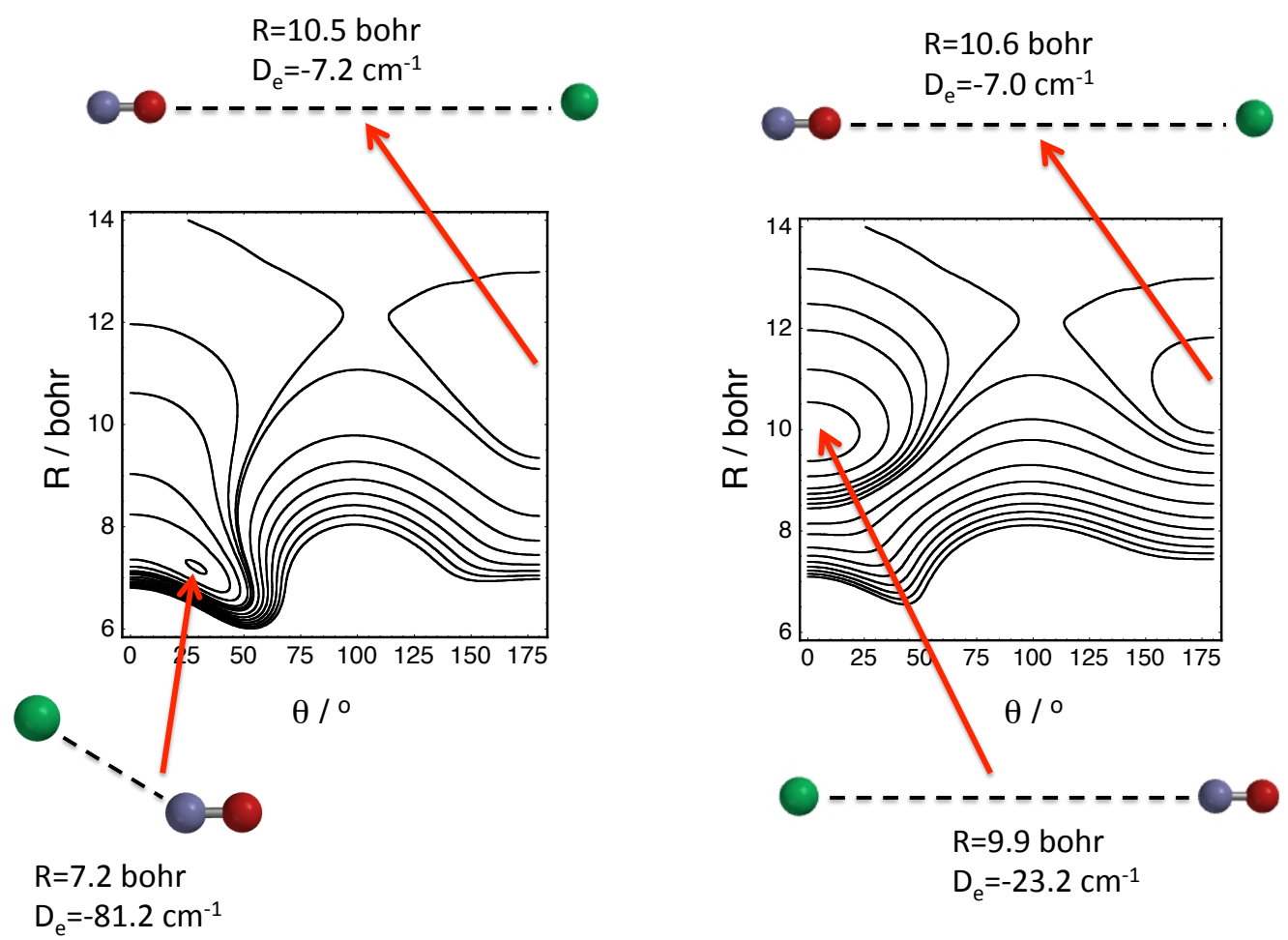